Famille d'extensions quartiques non galoisiennes et totalement imaginaires 


\title{
FAMILLE D'EXTENSIONS QUARTIQUES NON GALOISIENNES ET TOTALEMENT IMAGINAIRES
}

\author{
M. Vérant \\ 19 mars 1996
}

\begin{abstract}
Résumé
As in a previous article, we describe the ring of integers and the group of units of two families of non-Galois quartic extensions, one is given by the first coordinate of a torsion point of an elliptic curve and the other is the maximal real field contained in its Galois closure.

Résumé: Comme dans un article précédent, nous décrivons l'anneau des entiers et le groupe des unités de deux familles d'extensions quartiques non galoisiennes, une étant donnée par la première coordonnée d'un point de torsion d'une courbe elliptique, et l'autre étant le sous-corps réel maximal inclus dans sa clôture galoisienne.
\end{abstract}

\section{Construction.}

Considérons pour un nombre complexe $t \neq \pm 8$, la courbe elliptique d'équation:

$$
Y^{2}=X\left(4 X^{2}+t X+4\right) .
$$

Cette courbe est paramètrée par une fonction de Fueter $T$ associée à un réseau $\Omega$ de $\mathbb{C}$ et un point primitif de 4 -division $\psi$ de $\Omega$ et sa dérivée normalisée $T_{1}$ (la fonction de Weierstrass associée à $\Omega$ étant notée $\wp$, la fonction $T$ est définie par $T(z)=\frac{\wp(\psi)-\wp(2 \psi)}{\wp(z)-\wp(2 \psi)}$ (voir [2] pour plus de détails). 
Soit $\alpha \in \Omega$ tel que $2 \alpha=\psi$, puisque $T(\psi)=1$, d'après la formule de duplication, le nombre $T(\alpha)$ est racine du polynôme symétrique:

$$
P(X)=X^{4}-4 X^{3}-(t+2) X^{2}-4 X+1 .
$$

Remarquons que l'arithmétique des courbes elliptiques n'est plus utilisée dans ce qui suit.

Hypothèses: On suppose $t$ entier relatif, $t<-12$ et $\Delta=t^{2}-2^{6}$ sans facteur carré.

Nous allons étudier l'extension engendrée par une racine de $P(X)$, montrer qu'elle est quartique et totalement imaginaire (ceci grâce à l'hypothèse sur t), préciser la monogénéité de son anneau des entiers et vérifier que cette racine est une unité fondamentale. Nous en profitons pour étudier de même l'extension quartique réelle incluse dans sa clôture galoisienne et résoudre une équation de Thue correspondant au polynôme $P(X)$.

Je tiens à remercier Vincent Fleckinger, Jean Cougnard de leur aide précieuse et constante.

Il résulte de la proposition suivante que la famille des extensions étudiées n'est pas finie.

Proposition 1.1 L'ensemble des entiers naturels $t$ tels que $t^{2}-2^{6}$ est sans facteur carré n'est pas fini.

Démonstration: Nous raisonnons par l'absurde, en supposant qu'il existe un entier $s_{0}$ tel que, pour tout entier $s$, si $s \geq s_{0}$ alors $s$ ou $s+16$ a un facteur carré. Soit $s_{1} \in \mathbb{N}$ tel que $s_{1}>s_{0}$ et tel que $s_{1}-s_{0}$ soit un multiple de 4 . Notons $E$ l'ensemble des entiers $s$, sans facteur carré, tels que $16 s_{0}<s \leq 16 s_{1}$. Si $s$ appartient à $E$ alors $s$ n'est pas un multiple de 4 et $s+16$ a un facteur carré. $E$ a donc au plus $6\left(s_{1}-s_{0}\right)$ éléments, ce qui s'écrit encore, $\mu$ désignant la fonction de Mobiüs:

$$
\sum_{s=16 s_{0}+1}^{16 s_{1}}|\mu(s)| \leq 6\left(s_{1}-s_{0}\right) .
$$

D'après le théorème 334 de [5], il existe $A \in \mathbb{R}$ tel que pour tout $B \in \mathbb{N}$ on ait:

$$
\left|\sum_{s=1}^{B}\right| \mu(s)\left|-\frac{6 B}{\pi^{2}}\right|<A \sqrt{B} .
$$


Des deux inégalités précédentes, il résulte:

$$
\frac{6\left(16 s_{1}\right)}{\pi^{2}}-A \sqrt{16 s_{1}} \leq 16 s_{0}+6\left(s_{1}-s_{0}\right)
$$

ou encore

$$
\frac{1}{\pi^{2}}-\frac{1}{16} \leq \frac{10 s_{0}}{96 s_{1}}+\frac{A}{6 \sqrt{16 s_{1}}}
$$

cette inégalité étant valable quelque soit $s_{1}$, ce qui est absurde.

Notations: La partie réelle (resp. la partie imaginaire, le module, le conjugué) d'un nombre complexe $z$ est noté $\Re(z)$ (resp. $\Im(z),|z|, \bar{z}$ ).

Le polynôme aux inverses de $P(X)$ étant

(7) $P_{1}(X)=X^{2}-4 X-t-4=(X-2-i \sqrt{-t-8})(X-2+i \sqrt{-t-8})$,

notons

$$
\begin{gathered}
s=-t-8, y=2+i \sqrt{s}, u=\sqrt{\sqrt{1+\frac{16}{s}}+1} \\
\delta=\frac{1}{\sqrt{2}}\left(\frac{4}{u}+i u \sqrt{s}\right), x=\frac{1}{2}(y+\delta),
\end{gathered}
$$

On vérifie que $y^{2}-4=\delta^{2}$ et par conséquent d'une part on a $y=x+\frac{1}{x}$ et d'autre part $x$ est une racine commune des polynômes $X^{2}-y X+1$ et $P(X)$. Notons enfin:

$$
k=\mathbb{Q}(\sqrt{t+8}), k^{\prime}=\mathbb{Q}(\sqrt{t-8}), K=\mathbb{Q}(x) .
$$

On en déduit facilement les encadrements suivants:

$$
\begin{gathered}
\sqrt{2} \leq u \leq 2, \frac{3}{2} \leq \Re(x) \leq 2, \\
\frac{3}{2} \sqrt{s} \leq \Im(x) \leq 2 \sqrt{s},|x| \leq 2 \sqrt{1+s} .
\end{gathered}
$$

Une meilleure majoration de $\ln (|x|)$ est la suivante:

Lemme 1.1 On a $\ln (|x|) \leq \frac{1}{2} \ln (s)+\frac{3}{s}$ 
Démonstration: De (8) et (9) on déduit:

$$
\left\{\begin{aligned}
2|x| & \leq|y|+|\delta| \\
|y| & =\sqrt{4+s} \\
|\delta| & =\Delta^{\frac{1}{4}}=\sqrt{s} \sqrt[4]{1+\frac{16}{s}}
\end{aligned}\right.
$$

ou encore

$$
|x| \leq \frac{1}{2} \sqrt{s}\left(\sqrt{1+\frac{4}{s}}+\sqrt[4]{1+\frac{16}{s}}\right)
$$

On a facilement

$$
\sqrt{1+\frac{4}{s}} \leq 1+\frac{2}{s}, \sqrt[4]{1+\frac{16}{s}} \leq 1+\frac{4}{s}, \ln \left(1+\frac{3}{s}\right) \leq \frac{3}{s} .
$$

De (14) et (15), on déduit la majoration cherchée.

\section{Etude de $K$ et de sa clôture galoisienne $N$.}

Proposition 2.1 Le polynôme irréductible de $x$ sur $\mathbb{Q}$ est $P(X)$. L'extension $K$ est quartique, totalement imaginaire, contient $k$ et est égale à $\mathbb{Q}(\delta)$. De plus, $K$ n'est pas galoisienne et a pour clôture galoisienne $N=\mathbb{Q}(\delta, \bar{\delta})=$ $\mathbb{Q}(x, \sqrt{t-8})$ qui contient $k^{\prime}$.

\section{Démonstration:}

1) Au cours des notations du paragraphe précédent, nous avons vu que $x$ est une racine de $P(X)$. Le nombre $x-1$ est racine de

$$
P(X+1)=X^{4}+s X^{2}+2 s X+s
$$

polynôme d'Eisenstein en tout facteur premier de $s$ : l'extension $K$ est donc quartique et le polynôme irréductible de $x$ sur $\mathbb{Q}$ est $P(X)$. Le polynôme $P(X)$, étant symétrique, a pour racines $x, \frac{1}{x}, \bar{x}, \frac{1}{\bar{x}}$. L'élément $x$ n'étant pas réel, $K$ est totalement imaginaire.

2) Puisque $y=x+\frac{1}{x}$, on a $y \in K$ et donc $k$ est inclus dans $K$. D'après (7), on a $\delta^{2}=y^{2}-4=4 y+t$ et donc $y \in \mathbb{Q}(\delta)$; d'après $(9)$, on en déduit que $K=\mathbb{Q}(\delta)$.

3) L'extension $K$ n'est pas le compositum de $k$ et $k^{\prime}$ car tout facteur premier 
de $s$ se ramifie totalement dans $K$ mais a pour indice de ramification 2 dans $k k^{\prime}$; donc $\sqrt{t-8}$ n'appartient pas à $K$. Puisque $\delta \bar{\delta}=\sqrt{\Delta}$, il en résulte que $K$ n'est pas galoisienne et a pour clôture galoisienne

$$
N=\mathbb{Q}(x, \sqrt{t-8})=\mathbb{Q}(\delta, \bar{\delta}) .
$$

Proposition 2.2 L'extension réelle quartique $K^{\prime}$ incluse dans $N$ a pour éléments primitifs $\Re(x), \Re(\delta)$ et $|x|^{2}$. Le polynôme irréductible de $|x|^{2}$ est:

$$
R(X)=X^{4}+(t+4) X^{3}+2(t+11) X^{2}+(t+4) X+1 .
$$

Démonstration: D'après (1.9), $\Re(\delta)$ est racine de

$$
Q(X)=X^{4}+s X^{2}-4 s
$$

qui est un polynôme d'Eisenstein en tout facteur premier de $s$. Le polynôme $Q(X)$ est donc irréductible dans $\mathbb{Z}[X]$ et $\mathbb{Q}(\delta+\bar{\delta})$ est donc quartique. Puisque les racines de $P(X)$ sont $x, \frac{1}{x}, \bar{x}$ et $\frac{1}{\bar{x}}$ et ont pour somme 4 , on a:

$$
2=\Re(x)+\Re\left(\frac{1}{x}\right)=\Re(x)\left(1+\frac{1}{|x|^{2}}\right) .
$$

De plus, d'après (1.8) et (1.9) on a:

$$
1+\frac{\Re(\delta)}{2}=\Re(x)
$$

il résulte de $(20)$ et $(21)$ que $\Re(\delta)=\frac{2|x|^{2}-2}{|x|^{2}+1}$. Le polynôme $R(X)$ est obtenu à partir de $Q(X)$ en substituant $\frac{2 X-2}{X+1}$ à $X$.

Proposition 2.3 Le nombre $i|x|$ est un élément primitif de $N$, son polynôme irréductible sur $K$ est $X^{2}-(x-1) X+x$. 
Démonstration: On obtient l'égalité suivante

$$
(t-8) x^{2}=\left(x^{2}-6 x+1\right)(x+1)^{2},
$$

en considérant le polynôme réduit de $P(X)$ modulo 8 . On est naturellement conduit à utiliser le polynôme $X^{2}-(x-1) X+x$; il a pour racine

$$
z=\frac{1}{2}\left(x-1+\frac{x}{x+1} \sqrt{t-8}\right) \text {. }
$$

qui, comme $\sqrt{t-8}$, est un élément de $N$ qui n'appartient pas à $K$, d'après la proposition 2.1. On a donc $x=z \frac{z+1}{z-1}$ et un calcul montre que

$$
R\left(-X^{2}\right)=(X-1)^{4} P\left(X \frac{X+1}{X-1}\right)
$$

par conséquent $z^{2}$ est une racine du polynôme $R(-X)$. Le polynôme $R(X)$ a pour seules racines réelles $|x|^{2}$ et son inverse; un calcul (fastidieux) montre que $z$ vérifie $\Re(z)=0$ et $\Im(z)>1$. On en déduit l'égalité $z=i|x|$; le degré de $i|x|$ sur $K$ étant 2 et celui de $|x|^{2}$ sur $\mathbb{Q}$ étant 4 , le nombre $i|x|$ est un élément primitif de $N$.

Soient $\tau$ la conjugaison complexe et $\sigma$ un automorphisme de $N$ tel que $\sigma(\delta)=-\bar{\delta}$; le schéma ci-dessous récapitule les sous-corps $L$ de $N$ et pour chacun d'entre eux, le groupe de galois de $N / L$ :

$N$

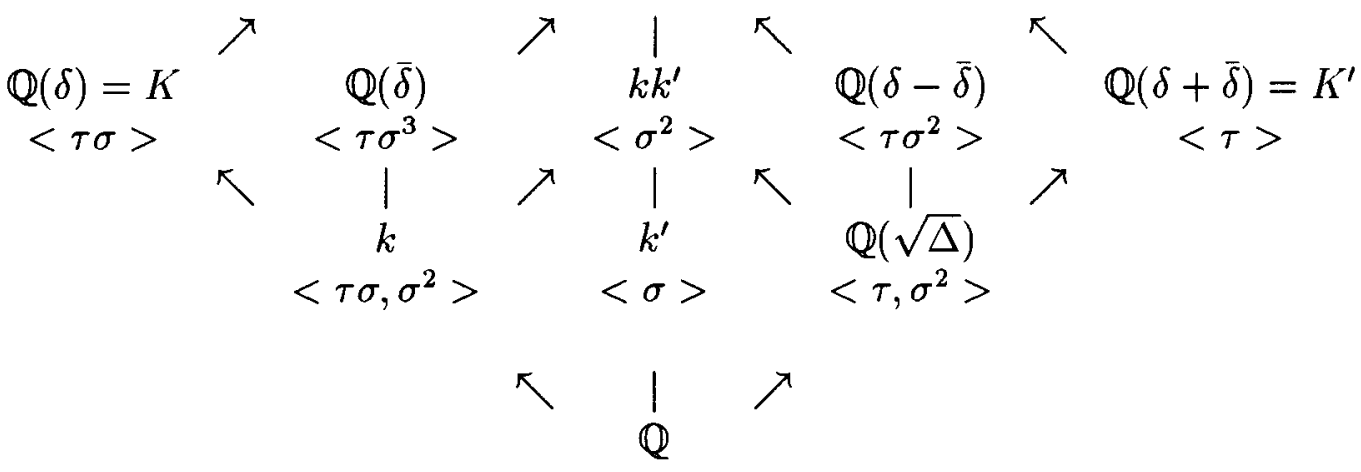




\section{$3 \quad$ Anneau des entiers de $K$ et de $N$.}

Notons $d_{K}$ le discriminant de $K$ et $d_{N}$ le discriminant de $N$.

\section{Proposition 3.1}

1) Si $t \not \equiv 1 \bmod 4$ alors l'anneau $O_{K}$ des entiers de $K$ est monogène et engendré par $x$ et $d_{K}=4^{2}(t-8)(t+8)^{3}$.

2) Si $t \equiv 1 \bmod 4$ alors $O_{K}$ a pour base

$$
\mathfrak{B}=\left\{1, \frac{y-1}{2}, x, \frac{x(y-1)}{2}\right\},
$$

$d_{K}=(t-8)(t+8)^{3}$ et de plus 2 est un diviseur commun inessentiel $d u$ discriminant (d.c.i.d.) de $K$.

Démonstration: Le discriminant de $P(X)$ est $d=4^{2}(t-8)(t+8)^{3}$ et on a vu que tout facteur premier de $s$ est ramifié totalement et modérément dans $K$.

1) Si $t \not \equiv 1 \bmod 4$ alors 2 est ramifié dans $k$ qui a pour discriminant $4(t+8)$ et on déduit que $d$ est le discriminant de $K$.

2) Supposons $t \equiv 1 \bmod 4$. Le polynôme irréductible de $\frac{y-1}{2}$ est

$$
X^{2}-X-\frac{t+7}{4}
$$

le nombre $\frac{y-1}{2}$ est donc un entier, la base $\mathfrak{B}$ est entière, a pour discriminant $(t-8)(t+8)^{3}$ et elle engendre donc $O_{K}$.

Montrons que 2 est un d.c.i.d. de $K$ et pour cela, étudions sa décomposition dans $K$. L'entier 2 est décomposé dans $k$. La décomposition en produit de facteurs premiers du polynôme réduit de $P(X)$ dans $\mathbb{Z}[X] / 2 \mathbb{Z}[X]$ est

$$
\left(X^{2}+X+\overline{1}\right)^{2}
$$

par conséquent $P(X)$ n'a pas de racine dans $\mathbb{Q}_{2}$ et par suite les idéaux premiers divisant 2 dans $k$ sont inertes dans $K$. On utilise alors un critère de Hasse ([4] ch.25): il y a deux idéaux premiers de $K$ divisant 2, de degré résiduel 2 et, par ailleurs, $\mu$ désignant la fonction de Möbius,

$$
\pi(2)=\frac{1}{2} \sum_{d \mid 2} \mu\left(\frac{2}{d}\right) 2^{d}=1,
$$


on en déduit que 2 est un d.c.i.d. de $K$.

Proposition 3.2 L'anneau des entiers de $N$ est $O_{K}$-monogène et engendré par $i|x|$; le discriminant de $N$ est $d_{N}=d_{K}^{2}(t-8)^{2}$.

Démonstration: 1) Soit $p$ un diviseur premier de $t-8$. La décomposition en produit de facteurs premiers du polynôme réduit de $P(X)$ dans $\mathbb{Z}[X] / p \mathbb{Z}[X]$ est

$$
\left(X^{2}-\overline{6} X+\overline{1}\right)(X+\overline{1})^{2}
$$

d'après la proposition précédente, il existe donc deux idéaux premiers $\mathfrak{P}_{1}$ et $\mathfrak{P}_{2}$ de $K$ tels que

$$
p O_{K}=\mathfrak{P}_{1} \mathfrak{P}_{2}^{2}
$$

de plus $\mathfrak{P}_{1}$ a pour degré résiduel 2 sur $\mathbb{Z}$ et est ramifié dans $N$ et $\mathfrak{P}_{2}$ est inerte dans $N$.

2) Le discriminant du polynôme irréductible sur $K$ de $i|x|$ est égal à $x^{2}-$ $6 x+1$ d'après la proposition 2.2 ; on vérifie par le calcul que $x^{2}-6 x+1$ a pour norme $(t-8)^{2}$ sur $\mathbb{Q}$; on a donc bien $O_{N}=O_{K}[i|x|]$, et $d_{N}=d_{K}^{2}(t-8)^{2}$ en utilisant la formule de composition des discriminants.

Remarque: Supposons $t \equiv 1$ modulo 4 . Suivant une suggestion de Jean Cougnard nous avons cherché, en vain, une base normale de $N / k$. Signalons toutefois que les éléments $x_{1}=x-\frac{y-1}{2}$ et $z_{1}=i|x|+x \frac{y-1}{2}$ engendrent respectivement des bases normales de $K / k$ et de $N / K$; en effet la trace de $x_{1}$ sur $k$ est 1 , celle de $z_{1}$ sur $K$ est $x^{2}$ et de plus, d'après les propositions 3.1 et 3.2 , on a $O_{N}=O_{K}\left[z_{1}\right]$ et $O_{K}=O_{k}\left[x_{1}\right]$.

\section{Groupe des unités de $K$.}

Proposition 4.1 Le groupe des unités de $K$ est engendré par $x$ et -1 .

\section{Démonstration:}

1) Le sous-corps quadratique de $K$ est $\mathbb{Q}(\sqrt{t+8})$, l'entier $t+8$ est sans facteurs carrés et $t+8<-3$ donc le sous-groupe de torsion de $K$ est $\{ \pm 1\}$.

2) Puisque $K$ est totalement imaginaire, le rang du groupe des unités est 1 et $x$ étant une unité, le régulateur $R_{K}$ de $K$ est majoré par $2 \ln (|x|)$. D'après les résultats de Bergé et Martinet ([1]), puisque $\left|d_{K}\right| \notin\{392,605\}$, on a:

$$
R_{K} \geq \frac{1}{4} \ln \left(\frac{\left|d_{K}\right|}{16}\right) ;
$$


D'après la proposition précédente, on a $\left|d_{K}\right| \geq s^{4}$ et donc:

$$
\frac{1}{4} \ln \left(\frac{\left|d_{K}\right|}{16}\right) \geq \ln (s)-\ln (2) \text {. }
$$

D'après le lemme $1.2,(31)$ et $(32)$, on déduit alors:

$$
\frac{2 \ln (|x|)}{R_{K}}<2 .
$$

Si $x$ n'est pas une unité fondamentale, soit $v$ celle-ci; il existe $n \in \mathbb{N}, n>1$, tel que $|x|=|v|^{n}$, et on a alors $2 \ln (|x|)=n R_{K}$, ce qui contredit (33).

\section{Anneau des entiers de $K^{\prime}$.}

Notons $d_{K^{\prime}}$ le discriminant de $K^{\prime}$ et

$$
z_{t}=\left\{\begin{array}{cl}
\frac{1}{8}\left(-3+|x|^{6}\right) & \text { si } t \equiv 1 \bmod 8 \\
\frac{1}{4}\left(1+2|x|^{2}+2|x|^{4}+|x|^{6}\right) & \text { si } t \equiv 3 \bmod 4 \\
\frac{1}{8}\left(-3+4|x|^{2}+4|x|^{4}+|x|^{6}\right) & \text { si } t \equiv 5 \bmod 8
\end{array}\right.
$$

Proposition 5.1 L'anneau $O_{K^{\prime}}$ des entiers de $K^{\prime}$ a pour base

$$
\mathfrak{B}^{\prime}=\left\{1,|x|^{2},|x|^{4}, z_{t}\right\},
$$

et pour discriminant $d_{K^{\prime}}=\Delta^{2}(t+8)$ si $t \equiv 1 \bmod 4$ et $d_{K^{\prime}}=2^{2} \Delta^{2}(t+8)$ sinon.

Démonstration: Le polynôme $Q(X)$ introduit en (21) est d'Eisenstein en tous les facteurs premiers de $t+8$, donc ceux-ci se ramifient totalement et modérément dans $K^{\prime}$. Le discriminant de $R(X)$ est $d^{\prime}=2^{6} \Delta^{2}(t+8)$. De plus $K^{\prime}$ contient $\mathbb{Q}(\sqrt{\Delta})$, qui a pour discriminant $\Delta$. Donc $d_{K^{\prime}}$ et $d^{\prime}$ ont même valuation en tout facteur premier de $\Delta$. On vérifie directement que $z_{t}$ est un entier de $K^{\prime}$.

Pour terminer la démonstration, il suffit de vérifier que 2 est sauvagement ramifié dans $K^{\prime} / \mathbb{Q}(\sqrt{\Delta})$ si $t \equiv 3 \bmod 4$. Supposons donc qu'il existe $t^{\prime} \in \mathbb{Z}$ tel que $t=4 t^{\prime}+3$. Puisque $\Delta \equiv 1 \bmod 4$, l'entier 2 n'est pas ramifié dans $\mathbb{Q}(\sqrt{\Delta})$. Le polygone de Newton du polynôme

$$
Q(X+1)=X^{4}+4 X^{3}-\left(4 t^{\prime}+5\right) X^{2}-2\left(4 t^{\prime}+9\right) X+2\left(17+6 t^{\prime}\right)
$$

montre que par contre, 2 est ramifié dans $K^{\prime}$. 


\section{Groupe des unités de $K^{\prime}$.}

Lemme $6.1 \sqrt[3]{|x|^{2}} n^{\prime}$ appartient pas à $K^{\prime}$.

Démonstration: Raisonnons par l'absurde; il existe $a, b, c \in \mathbb{Z}$ tels que si on note:

$$
r(X)=X^{4}+a X^{3}+b X^{2}+c X+1,
$$

on ait $r(X) r(j X) r\left(j^{2} X\right)=R\left(X^{3}\right)$ avec $j=e^{\frac{2 i \pi}{3}}$.

On obtient le système:

$$
\left\{\begin{array}{ccc}
t+4 & = & a^{3}-3 a b+3 c \\
2 t+22 & = & b^{3}+3 a^{2}+3 c^{2}-3 b-3 a b c \\
t+4 & = & c^{3}-3 b c+3 a
\end{array}\right.
$$

Notons $a^{\prime}=a-1, c^{\prime}=c-1$ et $u=a^{\prime}-b+c^{\prime}$, on obtient le système:

$$
\left\{\begin{array}{c}
t=a^{3}+3 a^{2}+3 u-3 a^{\prime} b \\
\left(a^{\prime}-c^{\prime}\right)\left(a^{\prime 2}+c^{\prime 2}+3 u+a^{\prime} c^{\prime}\right)=0 \\
a^{\prime 3}-b^{3}+c^{\prime 3}+3 a^{\prime} b c^{\prime}=-16
\end{array}\right.
$$

a) Supposons que: $a^{\prime 2}+c^{\prime 2}+3 u+a^{\prime} c^{\prime}=0$. Du système précédent on déduit:

$$
\left\{\begin{array}{c}
\left(2 a^{\prime}+c^{\prime}\right)^{2}+3 c^{\prime 2}=-12 u, \\
u^{2}\left(u-3\left(a^{\prime}+c^{\prime}+3\right)\right)=-16 .
\end{array}\right.
$$

Par conséquent $u$ est un élément de $\{-1,-2,-4\}$.

L'équation $\alpha^{2}+3 \beta^{2}=-12 u$ a pour ensemble de solutions dans $\mathbb{Z}^{2}$ :

$$
\left\{\begin{array}{cl}
\{(0, \pm 1)\} & \text { si } u=-1 \\
\phi & \text { si } u=-2 \\
\{(0, \pm 4),( \pm 6, \pm 2)\} & \text { si } u=-4
\end{array}\right.
$$

On en déduit alors d'après (40) $u=-4, a^{\prime}=-2, c^{\prime}=-2$ et $t=-8$ ce qui est absurde.

b) Supposons que: $c^{\prime}=a^{\prime}$. Le système (39) devient:

$$
\left\{\begin{array}{c}
t=a^{3}+3 a^{\prime 2}-3 a^{\prime} b+6 a^{\prime}-3 b \\
\left(b+a^{\prime}\right)^{2}\left(b-2 a^{\prime}\right)=16
\end{array}\right.
$$

d'où on déduit $\left(a^{\prime}, b\right) \in\{(1,3),(-5,6),(-2,0)\}$ et $t=-8$, ce qui est absurde. 
Proposition 6.1 Le groupe des unités relatives de $K^{\prime} / \mathbb{Q}(\sqrt{\Delta})$ est engendré par -1 et $|x|^{2}$ et le groupe des unités de $K^{\prime}$ est engendré par $-1, \varepsilon$ et $|x|^{2}$ ou $|x| \sqrt{\varepsilon}$, le nombre $\varepsilon$ étant l'unité fondamentale de $\mathbb{Q}(\sqrt{\Delta})$.

Démonstration: Notons $R_{K^{\prime}}$ (resp. $R_{\mathbb{Q}(\sqrt{\Delta})}$ ) le régulateur de $K^{\prime}$ (resp. $\mathbb{Q}(\sqrt{\Delta})$ ). D'après le théorème 5.9 de Bergé et Martinet $([1])$, on a:

$$
\frac{R_{K^{\prime}}}{R_{\mathbb{Q}(\sqrt{\Delta})}} \geq \ln \left(\frac{s}{16}\right)
$$

car l'indice de Hasse et la constante $C$ du théorème valent 1 et car la norme du discriminant relatif de $K^{\prime} / \mathbb{Q}(\sqrt{\Delta})$ est $s$. D'après le lemme 1.2 , on a:

$$
4 \ln (|x|) \leq 2\left(\ln (s)+\frac{6}{s}\right) .
$$

Soit $v$ l'unité fondamentale relative de $K^{\prime} / \mathbb{Q}(\sqrt{\Delta})$. Il existe $n \in \mathbb{N}^{*}$ tel que $|x|^{2}=v^{n}$ et il nous reste à montrer que $n=1$. Pour $t \geq-114$ on le vérifie au moyen des logiciels Kant. Si $t \leq-114$, alors on a:

$$
n=\frac{\frac{4 \ln (|x|)}{R_{K^{\prime}}}}{R_{\mathbb{Q}(\sqrt{\Delta})}} \leq \frac{2\left(\ln (s)+\frac{6}{s}\right)}{\ln (s)-4 \ln (2)}<5 ;
$$

$n$ est différent de 2 et 4 , car $|x| \notin K^{\prime}$ d'après la proposition 2.2 , et différent de 3 d'après le lemme précédent, $n$ est donc égal à 1 .

Déterminons maintenant le groupe des unités de $K^{\prime}$. Soit $\xi$ une unité positive de $K^{\prime}$. Il existe $m \in \mathbb{Z}$ et $a \in\{0,1\}$ tels que $N_{K^{\prime} / \mathbb{Q}(\sqrt{\Delta})}(\xi)=\varepsilon^{2 m+a}$. L'unité $\xi^{2} \varepsilon^{-2 m-a}$ étant relative, il existe $n \in \mathbb{Z}$ et $b \in\{0,1\}$ tels que $\xi^{2}=$ $|x|^{4 n+2 b} \varepsilon^{2 m+a}$ et par suite $\xi=|x|^{2 n} \varepsilon^{m}|x|^{b} \sqrt{\varepsilon}^{a}$. Pour terminer, remarquons que le couple $(a, b)$ est différent de $(0,1)$ car $|x| \notin K$, et de $(1,0)$ car l'extension relative $K^{\prime} / \mathbb{Q}(\sqrt{\Delta})$ est ramifiée en dehors de 2 .

\section{Références}

[1] A. -M. Bergé et J. Martinet, Sur les minorations géométriques des régulateurs. Séminaire de théorie des nombres de Paris 1987-1988 
[2] P. Cassou-Noguès et M. J. Taylor: Elliptic functions and rings of integers. Progress in Math $\mathrm{n}^{0} 66$. Birkhauser.

[3] V. Fleckinger et M.Vérant: Familles d'extensions quartiques non galoisiennes. J. Number Theory $54 \mathrm{n}^{2}$ (1995), 99-273.

[4] H.Hasse : Number Theory.

Springer-Verlag, 1980.

[5] G.H.Hardy and E.M.Wright:

An Introduction to the Theory of Numbers.

Oxford University Press, fifth edition 1979.

M. Vérant

U.A. au C.N.R.S. $\mathrm{n}^{\circ} 741$

25030 Besançon Cedex

France 